TRANSACTIONS OF THE

AMERICAN MATHEMATICAL SOCIETY

Volume 356, Number 11, Pages 4371-4382

S 0002-9947(04)03503-2

Article electronically published on February 27, 2004

\title{
3-MANIFOLDS THAT ADMIT KNOTTED SOLENOIDS AS ATTRACTORS
}

\author{
BOJU JIANG, YI NI, AND SHICHENG WANG
}

\begin{abstract}
Motivated by the study in Morse theory and Smale's work in dynamics, the following questions are studied and answered: (1) When does a 3-manifold admit an automorphism having a knotted Smale solenoid as an attractor? (2) When does a 3-manifold admit an automorphism whose nonwandering set consists of Smale solenoids? The result presents some intrinsic symmetries for a class of 3-manifolds.
\end{abstract}

\section{INTRODUCTION}

The solenoids were first defined in mathematics by Vietoris in 1927 for 2-adic case and by others later in general case, which can be presented either in an abstract way (inverse limit of self-coverings of circles) or in a geometric way (nested intersections of solid tori). The solenoids were introduced into dynamics by Smale as hyperbolic attractors in his celebrated paper $[\mathrm{S}]$.

Standard notions in dynamics and in 3-manifold topology will be given in Section 2 The new definitions are the following:

Let $N=S^{1} \times D^{2}$, where $S^{1}$ is the unit circle and $D^{2}$ is the unit disc. Both $S^{1}$ and $D^{2}$ admit "linear structures". Let $e: N \rightarrow N$ be a "linear", $D^{2}$-level-preserving embedding such that (a) $e\left(S^{1} \times *\right)$ is a $w$-string braid in $N$ for each $* \in D^{2}$, where $w>1$ in an integer; (b) for each $\theta \in S^{1}$, the radius of $e\left(\theta \times D^{2}\right)$ is $1 / w^{2}$.

Definition. Let $M$ be a 3-manifold and let $f: M \rightarrow M$ be a diffeomorphism. If there is a solid torus $N \subset M$ such that $f \mid N$ (resp. $\left.f^{-1} \mid N\right)$ conjugates $e: N \rightarrow N$ above, we call $S=\bigcap_{h=1}^{\infty} f^{h}(N)$ (resp. $S=\bigcap_{h=1}^{\infty} f^{-h}(N)$ ) a Smale solenoid, which is a hyperbolic attractor (resp. repeller, or negative attractor) of $f$, and we also say $M$ admits $S$ as a Smale solenoid attractor and $N$ is a defining solid torus of $S$.

Smale solenoid in the above definition carries more information than a solenoid as a topological space. It also carries the information of braiding of $e(N)$ in $N$ and the knotting and framing of $N$ in $M$, in addition to the information that it is a hyperbolic attractor of a diffeomorphism $f: M \rightarrow M$.

Say a Smale solenoid $S \subset M$ is trivial if the core of a defining solid torus $N$ bounds a disc in $M$, otherwise say $S$ is knotted.

Received by the editors February 20, 2003 and, in revised form, April 18, 2003.

2000 Mathematics Subject Classification. Primary 57N10, 58K05, 37E99, 37D45.

Key words and phrases. 3-manifolds, homeomorphisms, attractors, solenoids, lens spaces.

This work was partially supported by a MOSTC grant and a MOEC grant. 
Theorem 1. Suppose $M$ is a closed orientable 3-manifold. There is a diffeomorphism $f: M \rightarrow M$ such that the non-wandering set $\Omega(f)$ contains a knotted Smale solenoid IF and ONLY IF the manifold $M$ has a lens space $L(p, q)$, with $p \neq 0, \pm 1$, as a prime factor.

Theorem 2. Suppose $M$ is a closed orientable 3-manifold. There is a diffeomorphism $f: M \rightarrow M$ with the non-wandering set $\Omega(f)$ a union of finitely many Smale solenoids IF and ONLY IF the manifold $M$ is a lens space $L(p, q), p \neq 0$.

Moreover for the IF part, the $\Omega(f)$ can be chosen to be two explicit $(p+1)$-adic solenoids, $p+1 \neq 0, \pm 1$.

Corollary 1. The diffeomorphism $f$ constructed in the IF part of Theorem 2 is $\Omega$-stable, but is not structurally stable.

\section{Motivations of the results.}

(1) From Morse theory. Let $f: M \rightarrow R$ be a non-degenerate Morse function. Then the gradient vector field $\operatorname{grad} f$ is a dynamical system on $M$ with hyperbolic $\Omega(\operatorname{grad} f)$. An important aspect of Morse theory is to use the global information of the singularities of $f$, or equivalently, the information of $\Omega(\operatorname{grad} f)$, to provide topological information of the manifold $M$. The classical examples are: if $\Omega(\operatorname{grad} f)$ consists of two points, then $M$ is the sphere by Reeb in 1952 [R], and if $\Omega(\operatorname{grad} f)$ consists of three points, then $M$ is a projective plane like manifold of dimension 2, 4, 8 or 16 proved by Eells and Kuiper in 1961 [EK]. The ONLY IF part of Theorems 1 and 2 are results of this style.

(2) From dynamics of Smale's school. In [S], for a diffeomorphism $f: M \rightarrow M$, Smale introduced the Axiom A, the strong transversality condition and the no cycle condition for $\Omega(f)$. Important results in the dynamics school of Smale are the equivalences between those conditions and various stabilities. For an Axiom A system $f$, Smale proved (Spectral Decomposition Theorem) $\Omega(f)$ can be decomposed into the so-called basic sets. He posed several types of basic sets: (a) Zero dimensional ones such as isolated points and Smale Horse Shoe; (b) Anosov maps and maps derived from Anosov; (c) expansive ones such as Smale solenoids.

All those results and notions need examples to testify. Most known examples are local. It is natural to ask a global question where topology and dynamics interact: For which manifold $M$ is there an $f: M \rightarrow M$ such that all the basic sets of $\Omega(f)$ belong to a single type above?

There is no restriction when $\Omega(f)$ is zero dimensional. The answer to the question for Anosov map was given by Porteous in $1974[\mathrm{Po}$. The ONLY IF part of Theorem 2 gives an answer about Smale solenoids for 3-manifolds. The Corollary also provides 3-dimensional global examples to testify the notions of stability.

We would also like to point out that there are many nice results on the interplay of topology and dynamics, mostly for flows. See $[\mathrm{F}],[\mathrm{Su}]$ and $[\mathrm{T}]$ for examples.

(3) Searching symmetries of manifolds with stability. A manifold $M$ admitting a dynamics $f$ such that $\Omega(f)$ consists of two hyperbolic attractors presents a symmetry of the manifold with certain stability. The sphere, the simplest closed manifold, admits a hyperbolic dynamics $f$ such that $\Omega(f)$ consists of exactly two points, one is a source, and the other is a sink. The attractors in this example are the simplest in three senses: (1) The topology of the attractors are trivial, (2) the embedding 
of attractors into the manifolds are trivial, (3) the restriction of the dynamics $f$ on the attractors are trivial. The IF part of Theorem 2 and the Corollary show more manifolds with such symmetry when we consider more complicated attractors suitably embedded into the manifolds.

Indeed we believe that many more 3-manifolds admit such symmetries if we replace the Smale solenoid by its generalization, the so-called Smale-Williams solenoid W] (the name is suggested in [Pe]).

The structure of the paper. For the convenience of the readers from both dynamics and 3-manifold topology, we list the needed notions and facts in dynamics and in 3-manifold topology in Section 2 . Sections 3 , 4 and 5 are devoted respectively to the proofs of the ONLY IF parts of Theorems 1 and 2 , the IF parts of Theorems 1 and 2, and the Corollary. Most notions in dynamics mentioned in Section 2 are only used in Section 5. To the authors, the most interesting part of the paper is the discovery of the IF part of Theorem 2 and its explicit constructive proof. Since such an explicit constructive proof is difficult to generalize to the case of Smale-Williams solenoids, we wonder if there is an alternative proof for the IF part of Theorem 2

\section{Notions AND FACTS IN DYNAMICS AND IN 3-MANIFOLD TOPOLOGY}

From dynamics. Everything in this part can be found in [Ni], unless otherwise indicated.

Assume $f: M \rightarrow M$ is a diffeomorphism of a compact $n$-manifold $M$.

An invariant set of $f$ is a subset $\Lambda \subset M$ such that $f(\Lambda)=\Lambda$. A point $x \in M$ is non-wandering if for any neighborhood $U$ of $x, f^{n}(U) \cap U \neq \emptyset$ for infinitely many integers $n$. Then $\Omega(f)$, the non-wandering set of $f$, defined as the set of all nonwandering points, is an $f$-invariant closed set. A set $\Lambda \subset M$ is an attractor if there exists a closed neighborhood $U$ of $\Lambda$ such that $f(U) \subset \operatorname{Int} U, \Lambda=\bigcap_{h=1}^{\infty} f^{h}(U)$, and $\Lambda=\Omega(f \mid U)$.

Say $f$ is structurally stable if all diffeomorphisms $C^{1}$-close to $f$ are conjugate to $f$. Say $f$ is $\Omega$-stable if all diffeomorphisms $C^{1}$-close to $f$ preserve the structure of $\Omega(f)$.

A closed invariant set $\Lambda$ of $f$ is hyperbolic if there is a continuous $f$-invariant splitting of the tangent bundle $T M_{\Lambda}$ into stable and unstable bundles $E_{\Lambda}^{s} \oplus E_{\Lambda}^{u}$ with

$$
\begin{aligned}
\left\|D f^{m}(v)\right\| \leq C \lambda^{-m}\|v\| \quad \forall v \in E_{\Lambda}^{s}, \forall m>0, \\
\left\|D f^{-m}(v)\right\| \leq C \lambda^{-m}\|v\| \quad \forall v \in E_{\Lambda}^{u}, \forall m>0,
\end{aligned}
$$

for some fixed $C>0$ and $\lambda>1$.

The Axiom A. The diffeomorphism $f: M \rightarrow M$ satisfies Axiom A if (a) the non-wandering set $\Omega(f)$ is hyperbolic; and (b) the periodic points of $f$ are dense in $\Omega(f)$.

Spectral Decomposition Theorem. For $f: M \rightarrow M$ satisfying Axiom $A, \Omega(f)$ can be decomposed in a unique way into finitely many disjoint sets $B_{1}, \ldots, B_{k}$, so that each $B_{i}$ is closed, $f$-invariant and contains a dense $f$-orbit.

The $B_{i}$ in the decomposition above are usually referred to as basic sets. 
Stable Manifold Theorem. Suppose $\Omega(f)$ is hyperbolic. Then for each $x \in \Omega(f)$, the sets $W^{s}(x, f)=\left\{y \in M \mid \lim _{j \rightarrow \infty} d\left(f^{j}(y), f^{j}(x)\right)=0\right\}$ and $W^{u}(x, f)=\{y \in$ $\left.M \mid \lim _{j \rightarrow \infty} d\left(f^{-j}(y), f^{-j}(x)\right)=0\right\}$ are smooth, injective immersions of the $E_{x}^{s}$ and $E_{x}^{u}$, respectively. Moreover, they are tangent to $E_{x}^{s}$ and $E_{x}^{u}$ at $x$, respectively.

$W^{s}(x, f)$ and $W^{u}(x, f)$ in the theorem are known as the stable and unstable manifolds of $f$ at $x$.

The Strong Transversality Condition. For all $x, y \in \Omega(f)$, the stable and unstable manifolds $W^{s}(x, f)$ and $W^{u}(y, f)$ are transverse.

The no cycle condition. An $n$-cycle of the Axiom A system is a sequence of basic sets $\Omega_{0}, \Omega_{1}, \ldots, \Omega_{n}$ with $\Omega_{0}=\Omega_{n}$ and $\Omega_{i} \neq \Omega_{j}$ otherwise, and such that $W^{u}\left(\Omega_{i-1}\right) \cap W^{s}\left(\Omega_{i}\right) \neq \emptyset$. An Axiom A system satisfies the no-cycle condition if it has no $n$-cycle for all $n \geq 1$.

Stability theorem (See the survey paper [Ha]). (a) Axiom A and the strong transversality condition of $\Omega(f)$ are equivalent to the structural stability of $f$.

(b) Axiom $A$ and the no cycle condition of $\Omega(f)$ are equivalent to the $\Omega$-stability of $f$.

From 3-manifold theory. Everything in this part can be found in [He], unless otherwise indicated.

Let $M$ be a 3-manifold and $S$ an embedded 2-sphere separating $M$. Let $M_{1}$ and $M_{2}$ be the two 3-manifolds obtained by splitting $M$ along $S$ and capping-off the two resulting 2 -sphere boundary components by two 3 -cells. Then $M$ is a connected sum of $M_{1}$ and $M_{2}$, written $M_{1} \# M_{2}$.

A 3-manifold $M \neq S^{3}$ is prime if $M=M_{1} \# M_{2}$ implies one of $M_{1}, M_{2}$ is $S^{3}$.

Let $F$ be a connected compact 2-sided surface properly embedded in $M . F$ is said to be compressible if either $F$ bounds a 3-ball, or there is an essential, simple closed curve on $F$ which bounds a disk in $M$; otherwise, $F$ is said to be incompressible.

The following three results in 3-manifold topology are fundamental.

Kneser-Milnor's Prime Decomposition Theorem. Every closed orientable 3manifold $M \neq S^{3}$ can be expressed as a connected sum of a finite number of prime factors. Furthermore, the decomposition is unique up to order and homeomorphism.

Haken's Finiteness Theorem. Let $M$ be a compact orientable 3-manifold. Then the maximum number of pairwise disjoint, non-parallel, closed connected incompressible surfaces in $M$, denoted by $h(M)$, is a finite integer $\geq 0$.

Papakyriakopoulos's Loop Theorem. Let $M$ be a compact orientable 3-manifold and $S \subset M$ a closed orientable surface. If the homomorphism $i_{*}: \pi_{1}(S) \rightarrow$ $\pi_{1}(M)$ induced by the embedding $i: S \rightarrow M$ is not injective, then there is an embedded disc $D \subset M$ such that $D \cap S=\partial D$ and $\partial D$ is an essential circle in $S$.

For the definition of the lens space $L(p, q)$, see Section 4

\section{Proof of the ONLY IF parts of Theorems 1$]$ and 2}

We first prove the ONLY IF part of Theorem 1.

Proof. Suppose $f: M \rightarrow M$ has a knotted Smale solenoid $S$ as an attractor. Then $S=\bigcap_{h=1}^{\infty} f^{h}(N)$, and $\overline{M-N} \subset M-f(N)$, where $N$ is a defining solid torus of $S$. 
Since $f$ is a global homeomorphism, $\overline{M-N}$ and $\overline{M-f(N)}$ are homeomorphic. Suppose first that $\partial \overline{M-N}$ is an incompressible surface in $\overline{M-N}$. By Haken's Finiteness Theorem, $h(M)$, the maximum number of pairwise disjoint, non-parallel, closed incompressible surfaces in $M$, is a finite integer. Since the winding number $w$ of $f(N)$ in $N$ is $>1, \partial \overline{M-N}$ is incompressible in $\overline{N-f(N)}$ and is not parallel to $\partial \overline{M-f(N)}$. It follows that for any set $F$ of disjoint, non-parallel, incompressible surfaces of $\overline{M-N}, \partial \overline{M-f(N)} \cup F$ is a set of disjoint non-parallel closed incompressible surfaces in $\overline{M-f(N)}$. Hence $h(\overline{M-f(N)})$ is larger than $h(\overline{M-N})$, which contradicts the fact that $\overline{M-N}$ and $\overline{M-f(N)}$ are homeomorphic.

By the last paragraph, $\partial \overline{M-N}$ is compressible in $\overline{M-N}$. This means there is a properly embedded $\operatorname{disc}(D, \partial D) \subset(\overline{M-N}, \partial \overline{M-N})$ such that $\partial D$ is an essential circle in $\partial N$. Cutting $\overline{M-N}$ along $D$, we get a 3 -manifold, denoted by $M_{1}$, with $\partial M_{1}$ a 2 -sphere containing two copies $D_{1}$ and $D_{2}$ of $D$. Let $S_{*}$ be a boundary parallel 2-sphere in the interior of $M_{1}$. Now identifying $D_{1}$ and $D_{2}$, we get back to $\overline{M-N}$ and $S_{*}$ separates a punctured solid torus from $\overline{M-N}$; finally we glue back $N$ with $\overline{M-N}$ to get $M$ and $S_{*}$ separates a punctured lens space from $M$, i.e., $M$ contains a lens space $L$ as a prime factor.

If $L$ is $S^{3}$, then it is easy to see the core of $N$ bounds a disc, which contradicts the assumption that $S$ is knotted. If $L=S^{2} \times S^{1}$, then $N$ carries a generator $\alpha$ of $\pi_{1}\left(S^{2} \times S^{1}\right)=Z$. Since $f(N)$ is a $w$-string braid in $N$, we have $f_{*}(\alpha)=w \alpha$. Since $f$ is a homeomorphism, $f_{*}$ is an isomorphism. Hence $w=1$, and we reach a contradiction.

We have finished the proof of the ONLY IF part of Theorem 1

We are going to prove the ONLY IF part of Theorem 2

Suppose $\Omega(f)$ is a union of Smale solenoids $S_{1}, \ldots, S_{n}$. Then for each $i=1, \ldots, n$, it is known (more or less directly from the definition) that

(i) $f \mid S_{i}$ is hyperbolic and the periodic points of $f$ are dense in $S_{i}$;

(ii) $S_{i}$ is an $f$-invariant closed set and there is a dense $f$-orbit in $S_{i}$.

Then $f$ satisfies Axiom A by (i). By Spectral Decomposition Theorem, $\Omega(f)$ can be decomposed in a unique way into finitely many disjoint basic sets $B_{1}, \ldots, B_{k}$, so that each $B_{i}$ is closed, $f$-invariant and contains a dense $f$-orbit.

By (ii), each $S_{i} \subset B_{l}$ for some $l=1, \ldots, k$. Then from the facts that $S_{i}$ is an attractor of $f$ (or of $f^{-1}$ ) and that $B_{l}$ contains a dense $f$-orbit, there is a point $x \in \operatorname{Int} U_{i}$ so that its $f$-orbit $o(x)$ is dense in $B_{l}$, where $U_{i}$ is a closed neighborhood of $S_{i}$ mentioned in the definition of an attractor. Then it is clear that $x \in \Omega\left(f \mid U_{i}\right)$. Hence $x \in S_{i}$, thus $B_{l}=\overline{o(x)} \subset S_{i}$, so we must have $S_{i}=B_{l}$. Hence each $S_{i}$ is a basic set of $\Omega(f)$ and in particular, $\Omega(f)$ is a disjoint union of finitely many Smale solenoids.

Now the ONLY IF part of Theorem 2 follows from Lemma 1 and Lemma 2 below.

Lemma 1. Suppose $f: M \rightarrow M$ is a diffeomorphism and $\Omega(f)$ is a disjoint union of finitely many Smale solenoids. Then $\Omega(f)$ is a union of two solenoids, one is an attractor of $f$ and the other is an attractor of $f^{-1}$.

Proof. Suppose

$$
\Omega(f)=S_{1} \cup S_{2} \cup \ldots \cup S_{n}, \quad n \geq 1,
$$

is a disjoint union of solenoids, where either $S_{i}=\bigcap_{h=1}^{\infty} f^{h}\left(N_{i}\right)$ if $S_{i}$ is an attractor of $f$ or $S_{i}=\bigcap_{h=1}^{\infty} f^{-h}\left(N_{i}\right)$ if $S_{i}$ is an attractor of $f^{-1}$. Without loss of generality, 
we assume that the $N_{i}$ 's have been chosen so that $N_{i} \cap N_{j}=\emptyset$ if $i \neq j$ (since $S_{i} \cap S_{j}=\emptyset$ for $i \neq j$ ), and some $S_{i}$ is an attractor of $f$ (otherwise replace $f$ by $\left.f^{-1}\right)$. Then by re-indexing if necessary we assume that $S_{1}, \ldots, S_{k}$ are attractors of $f$ and the remaining $S_{j}$ are attractors of $f^{-1}$; henceforth, $k$ is the number of attracting solenoids. So we can assume

$$
f\left(N_{i}\right) \subset \operatorname{Int} N_{i}, \quad i=1, \ldots, k \leq n,
$$

and

$$
f^{-1}\left(N_{j}\right) \subset \operatorname{Int} N_{j}, \quad j=k+1, \ldots, n .
$$

For $i=1, \ldots, k$, let $V_{i}=\bigcup_{h=1}^{\infty} f^{-h}\left(\operatorname{Int} N_{i}\right)$. Since $f$ is a homeomorphism, $V_{i}$ is open. Moreover

$$
V_{i} \cap V_{j}=\emptyset, \quad 1 \leq i<j \leq k,
$$

and

$$
f\left(V_{i}\right)=V_{i},
$$

by the assumptions (2) and $N_{i} \cap N_{j}=\emptyset$ for $i \neq j$.

First we suppose $n=1$. Now Let $Y_{1}=M-\operatorname{Int} N_{1}$. Then $Y_{1}$ is compact and $f^{-1}\left(Y_{1}\right) \subset Y_{1}$ by $(2)$. Therefore $\Omega(f)=\Omega\left(f^{-1}\right)$ intersects $Y_{1}$, which contradicts (1).

So $n>1$. Suppose $k>1$. Let $Y_{2}=M-\bigcup_{j=k+1}^{n} S_{j}$. For each $i=1, \ldots, k$, $V_{i} \subset Y_{2} . \quad Y_{2}$ is connected, so it cannot be a disjoint union of $k>1$ open sets. Hence $Y_{3}=M-\left(\left(\bigcup_{j=k+1}^{n} S_{j}\right) \cup\left(\bigcup_{i=1}^{k} V_{i}\right)\right)$ is not empty. Suppose $x \in Y_{3}$. Since $S_{j}$ is compact, we can choose $N_{j}$ sufficiently small in order that $x \notin \bigcup_{j=k+1}^{n} \operatorname{Int} N_{j}$. Then $Y_{4}=M-\left(\left(\bigcup_{j=k+1}^{n} \operatorname{Int} N_{j}\right) \cup\left(\bigcup_{i=1}^{k} V_{i}\right)\right)$ is compact and is not empty. By (3), (5), we have $f\left(Y_{4}\right) \subset Y_{4}$. Hence $\Omega(f) \cap Y_{4} \neq \emptyset$, a contradiction.

We have proved that $f$ has exactly one attractor. By the same reason $f^{-1}$ also has exactly one attractor, therefore $n=2$ and the lemma is proved.

Lemma 2. Let $M$ be a closed orientable 3-manifold. If $f: M \rightarrow M$ is a diffeomorphism with $\Omega(f)$ a union of two disjoint Smale solenoids, then $M$ is a lens space and $M$ is not $S^{2} \times S^{1}$.

Proof. Suppose $\Omega(f)$ is a union of two disjoint solenoids $S_{1}$ and $S_{2}$. We may further assume that

$$
S_{1}=\bigcap_{h=1}^{\infty} f^{h}\left(N_{1}\right), \quad S_{2}=\bigcap_{h=1}^{\infty} f^{-h}\left(N_{2}\right), \quad N_{1} \cap N_{2}=\emptyset .
$$

We have $\bigcup_{h=1}^{\infty} f^{-h}\left(\operatorname{Int} N_{1}\right)=M-S_{2}$. It follows that

$$
f^{n}\left(\partial N_{2}\right) \subset \operatorname{Int} N_{1}, \quad M-N_{1} \subset f^{n}\left(N_{2}\right) \quad \text { for some large integer } n>1 .
$$

Since $H_{2}\left(N_{1}, Z\right)=0, \partial f^{n}\left(N_{2}\right)$ separates $N_{1}$ into two parts $Y^{\prime}$ and $Y^{\prime \prime}$ with $\partial Y^{\prime}=\partial f^{n}\left(N_{2}\right)$ and $\partial Y^{\prime \prime}$ has two components.

The homomorphism $i_{*}: \pi_{1}\left(\partial f^{n}\left(N_{2}\right)\right) \rightarrow \pi_{1}\left(N_{1}\right)$ induced by the embedding $i: \partial f^{n}\left(N_{2}\right) \rightarrow N_{1}$ is not injective, since $\pi_{1}\left(N_{1}\right)=Z$ and $\pi_{1}\left(f^{n}\left(\partial N_{2}\right)\right)=Z \oplus Z$. By the Loop Theorem, $\partial f^{n}\left(N_{2}\right)$ is compressible in $N_{1}$, that is, there is an embedded disc $D \subset N_{1}$ such that $D \cap \partial N_{2}=\partial D$ and $\partial D$ is an essential circle in $\partial f^{n}\left(N_{2}\right)$. Since the solid torus $N_{1}$ is irreducible, a standard argument shows that $\partial f^{n}\left(N_{2}\right)$ 

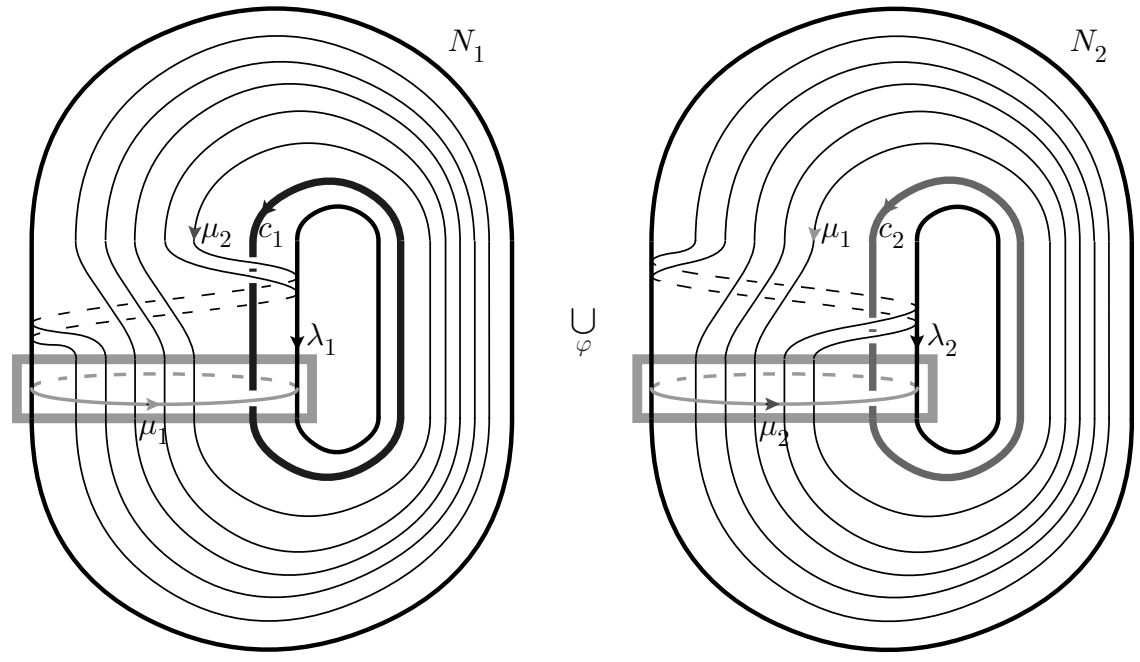

Figure 1. Lens space $L(p, q)$ as union of solid tori $N_{1} \cup_{\varphi} N_{2}$

bounds a solid torus $N^{\prime}$ in $N_{1}$, and therefore we have $N^{\prime}=Y^{\prime}$. Then by (7), we have

(8) $M=\left(M-N_{1}\right) \cup_{\partial N_{1}} N_{1}=M-N_{1} \cup_{\partial N_{1}} Y^{\prime \prime} \cup_{\partial f^{n}\left(N_{2}\right)} Y^{\prime}=f^{n}\left(N_{2}\right) \cup_{\partial f^{n}\left(N_{2}\right)} N^{\prime}$.

Hence $M$ is obtained by identifying two solid tori $f^{n}\left(N_{2}\right)$ and $N^{\prime}$ along their common boundary. So $M$ is a lens space.

Since $f$ is a homeomorphism, $N^{\prime \prime}=f^{-n}\left(N^{\prime}\right)$ is also a solid torus and $M$ is obtained by identifying two solid tori $N_{2}$ and $N^{\prime \prime}$ along their boundary. Now $f^{-1}\left(N_{2}\right)$ is a $w$-string braid in $N_{2}, w>1$. That $M$ is not $S^{2} \times S^{1}$ can be proved as before.

\section{Proof of the IF parts of Theorems 11 and 2}

Suppose $M$ is the lens space $L(p, q)$, where $p>0$ and $\operatorname{gcd}(p, q)=1$. Then $M$ is the union of two solid tori, $M=N_{1} \cup_{\varphi} N_{2}$, where the gluing map $\varphi: \partial N_{2} \rightarrow \partial N_{1}$ is an orientation reversing homeomorphism. On each torus $\partial N_{i}$, pick a meridianlongitude pair, denoted $\left\{\mu_{i}, \lambda_{i}\right\}$, as a basis of $H_{1}\left(\partial N_{i}\right)$. In $\partial N_{1}, \varphi\left(\mu_{2}\right)$ is the $(p, q)$-curve, that is $\varphi\left(\mu_{2}\right)=p \lambda_{1}+q \mu_{1}$, while $\varphi\left(\lambda_{2}\right)=r \lambda_{1}+s \mu_{1}$, with $p s-q r=1$. It is clear that in $\partial N_{1}$ we have $\varphi^{-1}\left(\mu_{1}\right)=p \lambda_{2}-r \mu_{2}$ and $\varphi^{-1}\left(\lambda_{1}\right)=-q \lambda_{2}+s \mu_{2}$. In Figure 1, the case of $M=L(5,2)$ and $\left(\begin{array}{cc}p & q \\ r & s\end{array}\right)=\left(\begin{array}{cc}5 & -2 \\ -2 & 1\end{array}\right)$ is shown as a concrete example.

Proof of the IF part of Theorem 2, The IF part of Theorem 2 is equivalent to the following

Claim. Suppose $M$ is a lens space $L(p, q), p>0$. Then there is a diffeomorphism $f: M \rightarrow M$ with $\Omega(f)$ a union of two $(p+1)$-adic solenoids, one is an attractor, the other is a repeller. 

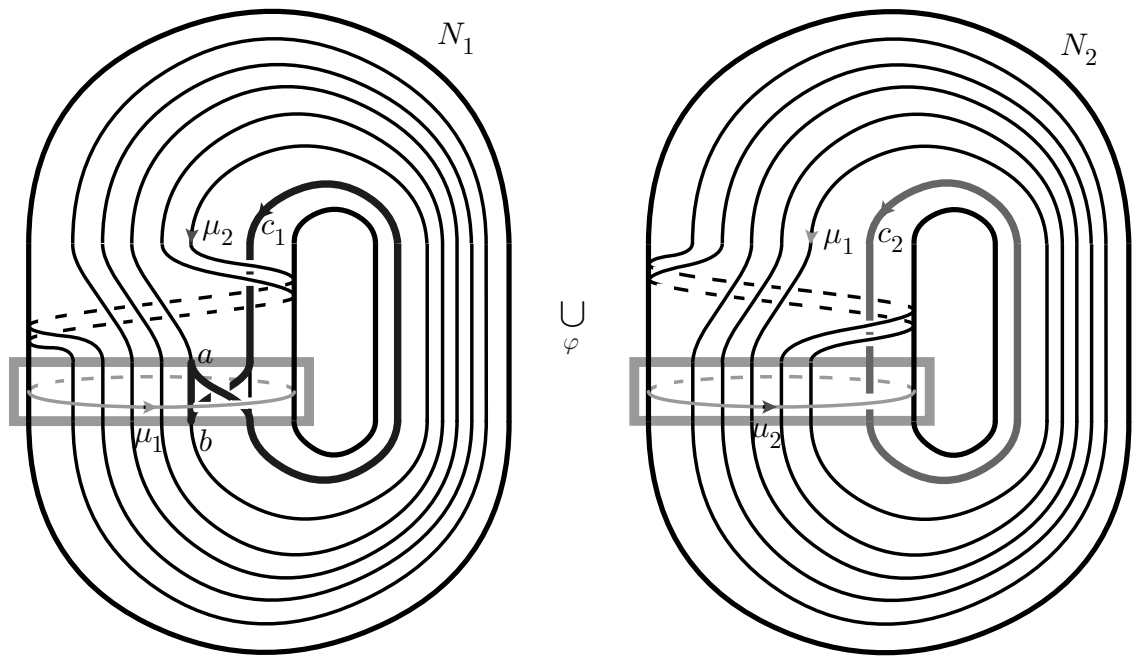

Figure 2. Writhe the core $c_{1}$ in $N_{1}$
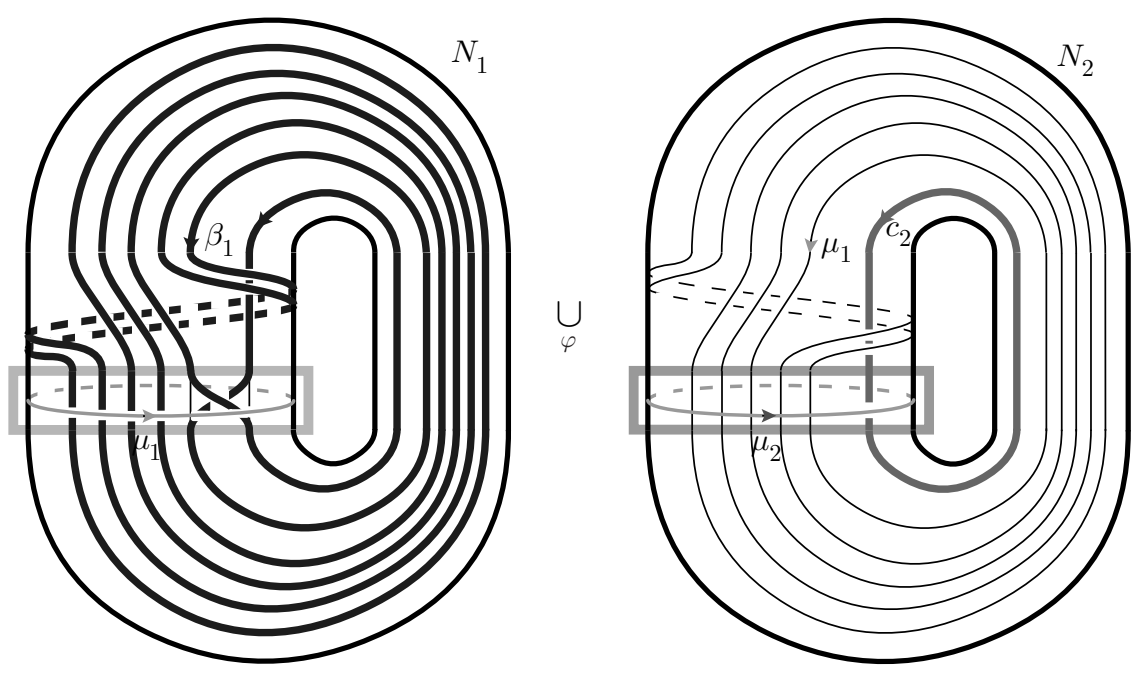

Figure 3. Closed braid $\beta_{1}$ in $N_{1}$ and core $c_{2}$ of $N_{2}$

We are going to prove this Claim.

Denote the oriented cores of $N_{1}, N_{2}$ by $c_{1}, c_{2}$ ( $c_{i}$ is homologous to $\lambda_{i}$ in $N_{i}$ ), respectively. We do the following operations to $c_{1}$, as indicated in Figure 2: Writhe $c_{1}$ locally, moving a subarc $\overline{a b}$ toward $\partial N_{1}$ and identify it with a subarc of $\varphi\left(\mu_{2}\right)$. Since $\mu_{2}$ bounds a meridian disk in $N_{2}$, we can push $\overline{a b}$ across the disk. The effect seen in $N_{1}$ is to replace $\overline{a b}$ with its complement in $\varphi\left(\mu_{2}\right)$; see Figure 2. Finally, pushing the obtained curve into Int $N_{1}$, we get a closed braid $\beta_{1}$ in $N_{1}$, as indicated in Figure 3. (In fact, $\beta_{1}$ is the "connected sum" of the "writhed" $c^{-1}$ with $\varphi\left(\mu_{2}\right)$ 

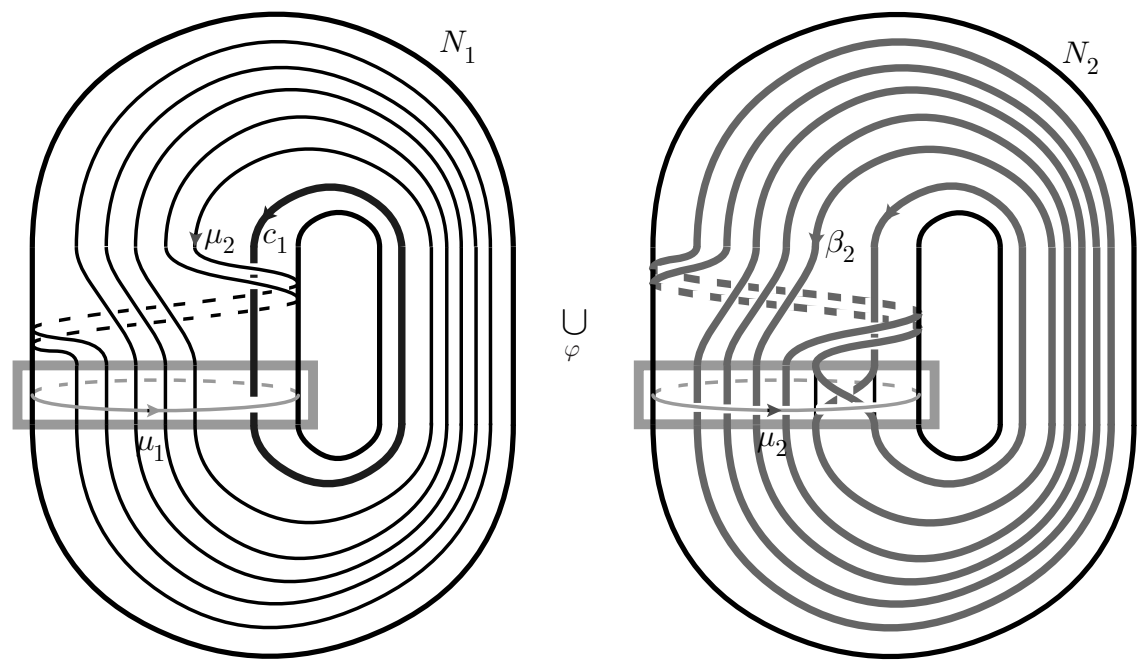

Figure 4. Core $c_{1}$ of $N_{1}$ and closed braid $\beta_{2}$ in $N_{2}$

in $N_{1}$.) Applying similar operations to $c_{2}$ in $N_{2}$, we get a closed braid $\beta_{2}$ in $N_{2}$, as indicated in Figure 4. Now $\beta_{1} \sqcup c_{2}$ and $c_{1} \sqcup \beta_{2}$ are two links in $M$.

Lemma 3. The two links $\beta_{1} \sqcup c_{2}$ and $c_{1} \sqcup \beta_{2}$ are isotopic in $M$.

Proof. Recall that $\beta_{1}$ is obtained by isotoping $c_{1}$; thus if we perform the inverse of the above isotopy, we can transform $\beta_{1}$ into $c_{1}$. We will show that the same isotopy also transforms $c_{2}$ into $\beta_{2}$.

From now on, we only use local pictures (represented in the rectangular frame in Figures 1-4) to show changes in both $N_{1}$ and $N_{2}$ simultaneously. The initial local picture of $\beta_{1} \sqcup c_{2}$ is shown in Figure 5-1. In $N_{1}$ (on the left), $\beta_{1}$ is a closed braid in $N_{1}$, and a segment of $c_{2}$ is shown outside of $\partial N_{1}$. On the right, most of $\beta_{1}$ coincides with $\mu_{2}$, along with the part slightly outside $\partial N_{2}$, and $c_{2}$ is the core of $N_{2}$. Our isotopy consists of the following three steps:

STEP 1. $\mu_{2}$ bounds a meridian disk in $N_{2}$, so we can pull $\beta_{1}$ across the disk. At the same time, a subarc of $c_{2}$ is pulled into $N_{1}$, as indicated in Figure 5-2.

STEP 2. In the local picture Figure $5-2, \beta_{1}$ has a self-crossing. A local half-twist will eliminate this self-crossing, as indicated in Figure 5-3. Take care so that a subarc $\overline{c d}$ of $c_{2}$ lies on $\varphi^{-1}\left(\mu_{1}\right)$. Now comparing Figure 5-2 and Figure 5-3, we find an interesting fact: except for the colors and labels, the left/right part of Figure 5-2 is the same as the right/left part of Figure 5-3. This symmetry suggests that the next step is a kind of inverse to Step 1.

Step 3. Push the subarc $\overline{c d}$ across the meridian disk of $N_{1}$, as indicated in Figure 5-4. We see that $\beta_{1}$ is deformed to $c_{1}$, and $c_{2}$ is deformed to $\beta_{2}$.

Proof of the Claim. Note that $\beta_{i}$ is a $(p+1)$-string braid in $N_{i}=S^{1} \times D_{i}^{2}$. Isotope $\beta_{i}$ in $N_{i}$ to meet all fiber discs $* \times D_{i}^{2}$ transversely. Let $\mathcal{N}(\gamma)$ denote the closed tubular neighborhood of a closed curve $\gamma$, and think of $N_{i}$ as $\mathcal{N}\left(c_{i}\right)$. 

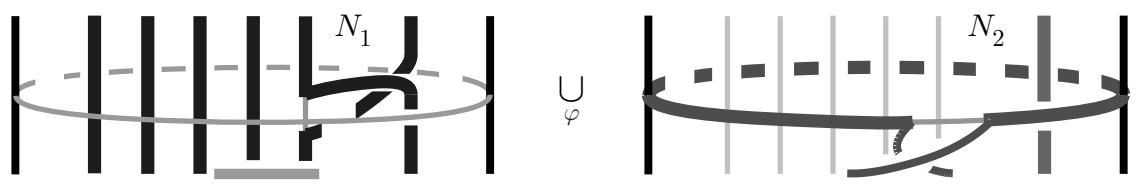

$\Downarrow$

Pull across the meridian disk of $N_{2}$
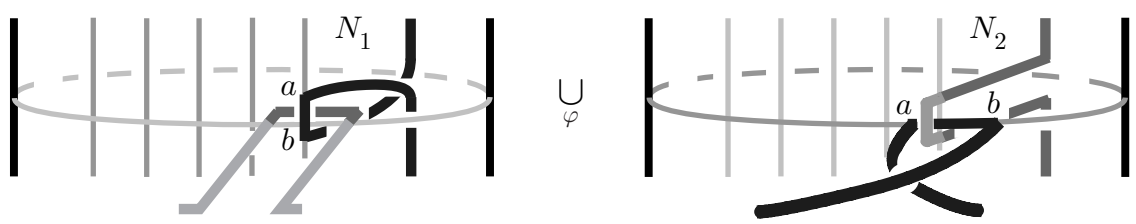

$\Downarrow$

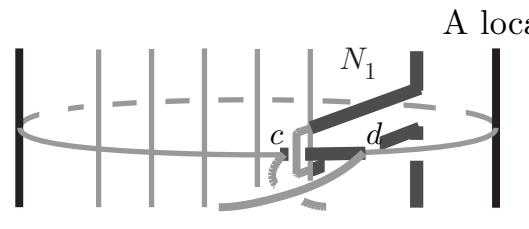

A local half twist

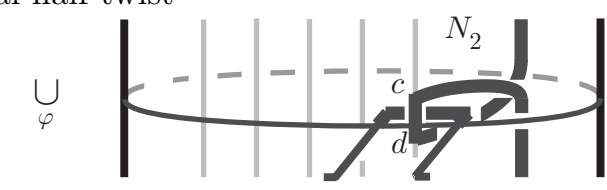

$\Downarrow$

Push across the meridian disk of $N_{1}$
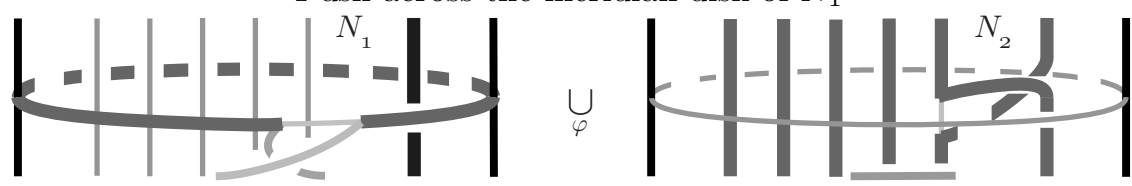

FiguRE 5. Local pictures of the 3-step isotopy from $\beta_{1} \sqcup c_{2}$ to $c_{1} \sqcup \beta_{2}$

Choose $\mathcal{N}\left(\beta_{i}\right)$ to be a disc bundle over $\beta_{i}$ embedded into $N_{i}$ so that each disc fiber $\subset * \times D^{2}$ (for $* \in S^{1}$ ) and has diameter $<1 /(p+1)^{2}$. Moreover we may assume that $N\left(\beta_{i}\right)$ misses the core $c_{i}$.

The isotopy provided by Lemma 3 that sends $\beta_{1} \sqcup c_{2}$ to $c_{1} \sqcup \beta_{2}$ can be adjusted to send $\mathcal{N}\left(\beta_{1}\right) \sqcup N_{2}$ to $N_{1} \sqcup \mathcal{N}\left(\beta_{2}\right)$, and to be "linear" and "disc-fiber preserving" on $\mathcal{N}\left(\beta_{1}\right) \sqcup N_{2}$. Then extend it to a diffeomorphism $f: M \rightarrow M$ which sends $\overline{N_{1}-N\left(\beta_{1}\right)}$ to $\overline{N_{2}-N\left(\beta_{2}\right)}$.

Now the $(p+1)$-adic solenoids $S_{1}=\bigcap_{h=1}^{\infty} f^{-h}\left(N_{1}\right)$ and $S_{2}=\bigcap_{h=1}^{\infty} f^{h}\left(N_{2}\right)$ are the repeller and the attractor of $f$, respectively. Moreover for each $x \notin S_{1} \cup S_{2}$, $f^{n}(x)$ approaches to $S_{2}$ as $n$ approaches to infinity, hence $\Omega(f)=S_{1} \cup S_{2}$.

We have finished the proof of the Claim, therefore the IF part of Theorem 2

Remark. By repeating the operations in the proof, we see that in the IF part of Theorem [2 the $\Omega(f)$ can be chosen to be two explicit $(m p+1)$-adic solenoids, $m p+1 \neq 0, \pm 1$.

Proof of the IF part of Theorem 1. Suppose $M=N \# L(p, q)$. It is easy to see that the isotopy above that sends $\beta_{1} \sqcup c_{2}$ to $c_{1} \sqcup \beta_{2}$ can be adjusted to send $N_{2}$ 
to $\mathcal{N}\left(\beta_{2}\right)$, to be "linear" and "disc-fiber preserving" on $N_{2}$, and to be the identity on a 3 -ball $B^{3}$ in $N_{1}$. Therefore there is a diffeomorphism on the $L(p, q)-\operatorname{int} B^{3}$ which has a knotted solenoid as a hyperbolic attractor and is the identity on its 2 -sphere boundary. Such a diffeomorphism can be extended to $M$ by the identity on the punctured $N$.

We have proved the IF part of Theorem 1

\section{Proof of Corollary 1}

We start from the end of the proof of the IF part of Theorem 2

Since $\Omega(f)$ consists of two Smale solenoids $S_{1}$ and $S_{2}, \Omega(f)$ meets Axiom A.

To prove the corollary, we need the following explicit description of stable and unstable manifolds of $\Omega(f)$.

First, $S_{1}$ is the union of stable manifolds of points in $S_{1}$, and $S_{2}$ is the union of unstable manifolds of points in $S_{2}$. Moreover, since $f^{-1} \mid N_{1}$ (resp. $f \mid N_{2}$ ) preserves the disc fibers of $N_{1}\left(\right.$ resp. $\left.N_{2}\right), \mathbb{F}_{1}=\bigcup f^{n}\left(S^{1} \times D_{1}\right)\left(\right.$ resp. $\left.\mathbb{F}_{2}=\bigcup f^{-n}\left(S^{1} \times D_{2}\right)\right)$ provides an $R^{2}$-foliation of $L(p, q)-S_{2}$ (resp. $R^{2}$-foliation of $\left.L(p, q)-S_{1}\right)$, which is the union of unstable manifolds of points in $S_{1}$ (resp. the union of stable manifolds of points in $S_{2}$ ). Hence we have

$$
W^{s}\left(S_{1}\right)=S_{1}, \quad W^{u}\left(S_{1}\right)=\mathbb{F}_{1}, \quad W^{u}\left(S_{2}\right)=S_{2}, \quad W^{s}\left(S_{2}\right)=\mathbb{F}_{2} .
$$

Therefore

$$
W^{s}\left(S_{1}\right) \cap W^{u}\left(S_{2}\right)=\emptyset, \quad W^{s}\left(S_{2}\right) \cap W^{u}\left(S_{1}\right) \neq \emptyset .
$$

It is clear that $f$ meets the no cycle condition. Hence $f$ is $\Omega$-stable by (b) of the Stability Theorem.

This $f$ is not structurally stable by the Stability Theorem (a) and the following Lemma 4.

Lemma 4. $\mathbb{F}_{1}$ and $\mathbb{F}_{2}$ do not meet transversely.

Proof. We need only to prove that $\mathbb{F}_{1} \mid$ and $\mathbb{F}_{2} \mid$, the restrictions of $\mathbb{F}_{1}$ and $\mathbb{F}_{2}$ on $\overline{N_{1}-N\left(\beta_{1}\right)}$ respectively, do not meet transversely.

Note that $\overline{N_{1}-N\left(\beta_{1}\right)}$ has two different $(p+1)$-punctured disc bundle structures provided by $\mathbb{F}_{1} \mid$ and $\mathbb{F}_{2} \mid$. (An $n$-punctured disc is obtained from the 2 -sphere by removing the interior of $n+1$ disjoint sub-discs.) More directly, one $(p+1)$ punctured disc bundle structure is induced from the pair $\left(N_{1}, N\left(\beta_{1}\right)\right)$ and the other is induced from the pair $\left(\overline{N_{1}-N\left(\beta_{1}\right)} \cup N_{2}, N_{2}\right)=\left(f^{-1}\left(N_{2}\right), f^{-1}\left(N\left(\beta_{2}\right)\right) \cong\right.$ $\left(N_{2}, N\left(\beta_{2}\right)\right)$.

It is easy to see that the restrictions of two fibrations $\mathbb{F}_{1} \mid$ and $\mathbb{F}_{2} \mid$ on $\overline{N_{1}-N\left(\beta_{1}\right)}$ meet transversely on $\partial \overline{N_{1}-N\left(\beta_{1}\right)}$.

Let $F_{1}$ be a fiber of $\mathbb{F}_{1} \mid$, which is a $(p+1)$-punctured disc. Suppose $\mathbb{F}_{1} \mid$ and $\mathbb{F}_{2} \mid$ meet transversely on $\overline{N_{1}-N\left(\beta_{1}\right)}$. Then the intersections of $F_{1}$ and $\mathbb{F}_{2} \mid$ provide a codimension one foliation on $F_{1}$ which meets $\partial F_{1}$ transversely. Now the genus $(p+1)$ closed surface $D\left(F_{1}\right)$, the double of $F_{1}$, will admit a codimension one foliation, which is impossible since $|p+1|>1$.

We have completed the proof of Corollary 1. 


\section{ACKNOWLEDGMENT}

We would like to thank the referee for his suggestions which enhanced the paper. We also thank Professors H. Duan, S. Gan and L. Wen for helpful conversations.

\section{REFERENCES}

[EK] J. Eells, Jr., and N.H. Kuiper, Manifolds which are like projective planes, IHES Publ. Math. 14(1962), 5-46. MR 26:3075

[F] J. M. Franks, Knots, links and symbolic dynamics, Ann. of Math. 113(1981), 529-552. MR 83h:58074

[Ha] S. Hayashi, Hyperbolicity, stability, and creation of homoclinic points, Proceedings of the International Congress of Mathematicians, vol. II (Berlin, 1998). Doc. Math. 1998, Extra vol. II, 789-796 (electronic). MR 99f:58115

[He] J. Hempel, 3-manifolds, Ann. of Math. Studies vol. 86, Princeton University Press, 1976. MR 54:3702

[Ni] Z. Nitecki, Differentiable dynamics, An introduction to the orbit structure of diffeomorphisms, MIT Press, 1971. MR 58:31210

[Pe] Y. B. Pesin, Dimension theory in dynamical systems, Contemporary views and applications, University of Chicago Press, 1997. MR 99b:58003

[Po] H. L. Porteous, Anosov diffeomorphisms of flat manifolds, Topology 11(1972), 307-315. MR 45:6035

[R] G. Reeb, Sur certaines propriétés topologiques des variétés feuilletées, Publ. Inst. Math. Univ. Strasbourg 11, pp. 5-89, 155-156. Actualités Sci. Ind., no. 1183, Hermann \& Cie., 1952. MR 14:1113a

[S] S. Smale, Differentiable dynamical systems, Bull. AMS 73(1967), 747-817. MR 37:3598

[Su] M. C. Sullivan, Visually building Smale flows in $S^{3}$, Topology Appl. 106(2000), 1-19. MR 2001g:37025

[T] E. S. Thomas, Jr., One-dimensional minimal sets, Topology 12(1973), 233-242. MR 48:5123

[W] R. F. Williams, One-dimensional non-wandering sets, Topology 6(1967), 473-487. MR 36:897

Department of Mathematics, Peking University, Beijing 100871, People's Republic of CHINA

E-mail address: jiangbj@math.pku.edu.cn

Department of Mathematics, Peking University, Beijing 100871, People's Republic of CHINA

Current address: Department of Mathematics, Princeton University, Princeton, New Jersey 08544

E-mail address: yni@princeton.edu

Department of Mathematics, Peking University, Beijing 100871, People's Republic of CHINA

E-mail address: wangsc@math.pku.edu.cn 